\title{
Improved staggered eigenvalues and epsilon regime universality in SU(2).
}

\section{E. Follana}

SUPA, Department of Physics and Astronomy, University of Glasgow, Glasgow G12 8QQ, U.K.

\author{
A. Hart* \\ SUPA, School of Physics, University of Edinburgh, King's Buildings, Edinburgh EH9 3JZ, U.K. \\ E-mail: a.harteed.ac.uk
}

\section{C.T.H. Davies}

SUPA, Department of Physics and Astronomy, University of Glasgow, Glasgow G12 8QQ, U.K.

\section{UKQCD and HPQCD Collaborations}

\begin{abstract}
We study the low-lying modes of staggered Dirac operators for quenched SU(2) and show that improvement changes the distribution from lattice-like to continuum-like at lattice spacings representative of current dynamical SU(3) simulations.

Epsilon regime universality predicts different distributions for the low-lying eigenvalues of the continuum and lattice staggered Dirac operators. At lattice spacings around $0.07 \mathrm{fm}$ we show that improved staggered eigenvalues have the continuum distribution (as predicted by the chiral Orthogonal Ensemble of random matrices), whilst unimproved fall on the discrete distribution (as per the chiral Symplectic Ensemble). The crossover is much more rapid than for SU(3).

In addition, improved staggered fermions give a good approximation to the Atiyah-Singer index theorem, appear to satisfy the Banks-Casher relation and show clear taste-degeneracy for the nonzero modes. All this indicates that taste-changing interactions are well under control at lattice spacings $0.07-0.13 \mathrm{fm}$, matching our findings for $\mathrm{SU}(3)$.
\end{abstract}

XXIVth International Symposium on Lattice Field Theory

July 23-28, 2006

Tucson, Arizona, USA

\footnotetext{
* Speaker.
} 


\begin{tabular}{ccccccc}
\hline$\beta$ & $L$ & \multicolumn{2}{c}{$a \sqrt{\sigma}$} & $a / \mathrm{fm}$ & $a L / \mathrm{fm}$ & label \\
\hline 1.22 & 12 & 0.309 & $(6)$ & 0.138 & 1.7 & "coarse" \\
1.36 & 16 & 0.219 & $(3)$ & 0.098 & 1.6 & "fine" \\
1.49 & 20 & 0.151 & $(3)$ & 0.068 & 1.4 & "super-fine" \\
1.57 & 28 & 0.116 & $(3)$ & 0.052 & 1.5 & "ultra-fine" \\
\hline
\end{tabular}

Table 1: The simulation parameters, with scale set by the string tensions, $\sqrt{K} \equiv a \sqrt{\sigma}$, given in Ref. [7]. "Physical" units come from assuming that $\sigma=(440 \mathrm{MeV})^{2}$. The scale at $\beta=1.57$ is a preliminary estimate.

\section{Introduction}

The low energy regime of Quantum Chromodynamics (QCD) exhibits a rich and interesting phenomenology, including the $U_{A}(1)$ axial anomaly, chiral symmetry breaking and the topological properties of the theory. A crucial step in elucidating these effects is understanding the low-lying eigenvalue spectrum of the Dirac operator. There are a number of detailed predictions of the properties of these low-lying modes, such as the existence of an Index Theorem, the Banks-Casher relation and the distribution of the first few eigenvalues in fixed topological charge sectors. Such effects are, however, inherently non-perturbative and can only be studied fully using techniques like lattice Monte Carlo simulation.

It is essential that the deformations to the eigenvalue spectrum brought about by the discretisation are small and that the fermions respond correctly to the gluonic topological charge. In an earlier study we showed that this was the case for improved staggered fermions on a quenched $\mathrm{SU}(3)$ gauge background $[1,2,3,4,5]$.

In this study, we turn our attention to the $\mathrm{SU}(2)$ gauge group. There are a number of reasons for doing this. Firstly, SU(2) is phenomenologically very similar to SU(3) and shares many of the same non-perturbative features including confinement and chiral symmetry breaking. In addition, if we equate the scales in the theories, we find remarkably close agreement for a wide range of physical parameters [6]. Finally, the $\varepsilon$-regime eigenvalue distribution predictions are markedly different in $\mathrm{SU}(2)$, allowing us to observe chiral symmetry restoration much more clearly than in SU(3). This has not, to our knowledge, been previously seen for staggered fermions.

\section{The simulations}

We begin by briefly describing our methodology. We have generated four quenched SU(2) gauge ensembles using a gauge action that is tree level Symanzik- and tadpole-improved:

$$
S=-\beta \sum_{x, \mu<v}\left\{\frac{5}{3} P_{\mu v}-\frac{1}{12} \frac{R_{\mu \mu \nu}}{u^{2}}-\frac{1}{12} \frac{R_{\mu v v}}{u^{2}}\right\} .
$$

$P, R$ are plaquettes and $2 \times 1$ loops respectively. The tadpole improvement coefficient $u$ is defined as the fourth root of the mean plaquette. The leading order discretisation errors are expected to be $\mathscr{O}\left(\alpha_{s} a^{2}\right)$.

The simulation parameters are given in Table 1. Aside from minor radiative corrections, this gauge action is identical to that used by the MILC and UKQCD Collaborations to generate SU(3) 

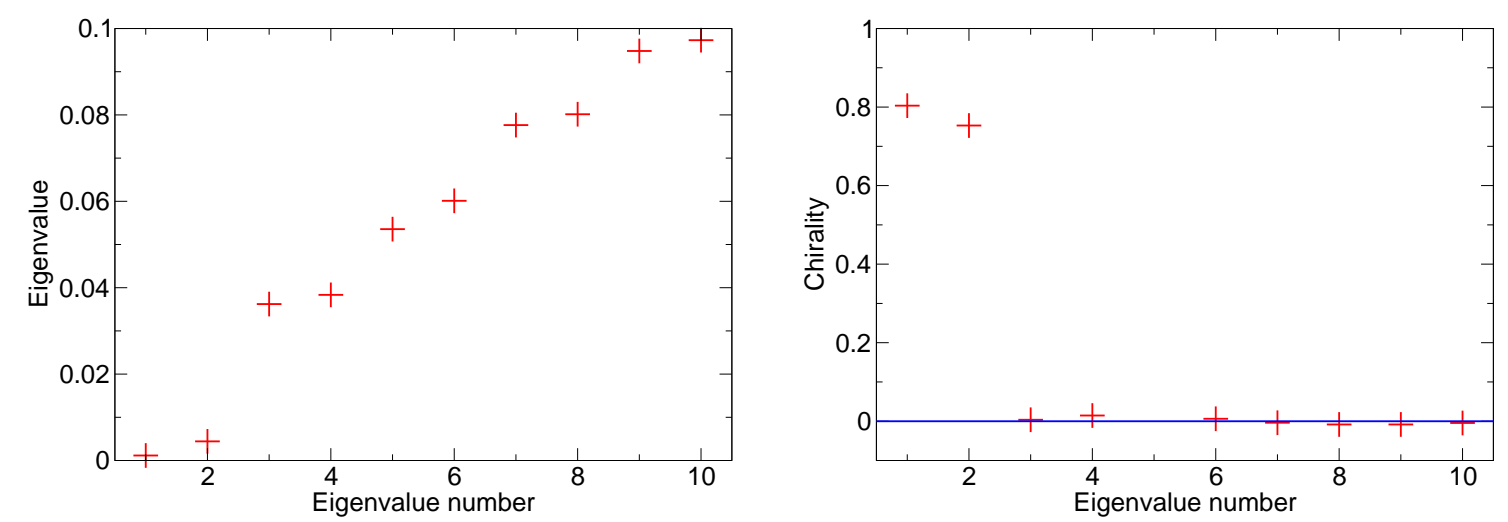

Figure 1: The low-lying HISQ spectrum on a typical super-fine lattice with $Q=2$. The left-hand panel shows the first 10 (unpaired, positive) eigenvalues, with their corresponding chirality on the right.

ensembles with $2+1$ dynamical quark flavours. We therefore use a similar labeling convention based on lattice spacing [8].

We measure the topological charge of each configuration by cooling the gauge fields using the 5-Loop improved (5Li) action [9, 10], and then applying the similarly improved topological charge operator. After 30 cooling sweeps the topological charge measurements are stable, and we obtain a continuum limit estimate of the topological susceptibility $\chi / \sigma^{2}=0.465$ (14) from the coarse, fine and super-fine ensembles. This compares well with the Wilson gauge action measurements summarised in Ref. [11].

\subsection{The fermion actions}

We have studied the spectrum of three variants of the staggered operator. The first, ONELINK, is the unimproved version. We also use the ASQTAD operator [12], which incorporates fattened gauge links and a Naik term to suppress taste-changing interactions. The sea quarks are described using the ASQTAD operator in the MILC/UKQCD dynamical simulations. Finally, we studied a more highly improved staggered operator that further reduces taste-changing, known as HISQ [13].

\subsection{Spectral features}

A single, continuum fermion species is described by the massless, gauge covariant Dirac operator, which is anti-Hermitian. The Dirac operator also anticommutes with $\gamma_{5},\left\{\mathbb{D}, \gamma_{5}\right\}=0$, implying that the eigenvalue spectrum is purely imaginary and symmetric about zero:

$$
\operatorname{sp}(\mathbb{D})=\left\{ \pm i \lambda_{s}, \lambda_{s} \in \mathbb{R}\right\}
$$

If $Q$ is the topological charge of the background $\mathrm{SU}(\mathrm{N})$ gauge field, the Atiyah-Singer theorem predicts there to be at least $Q$ zero-eigenvalue modes with chirality \pm 1 . The remaining, non-zero modes have zero chirality.

On the lattice, the massless staggered Dirac operator is anti-Hermitian and anticommutes with an operator $\varepsilon(x)$. The spectrum is therefore also pure imaginary and symmetric about zero. The 


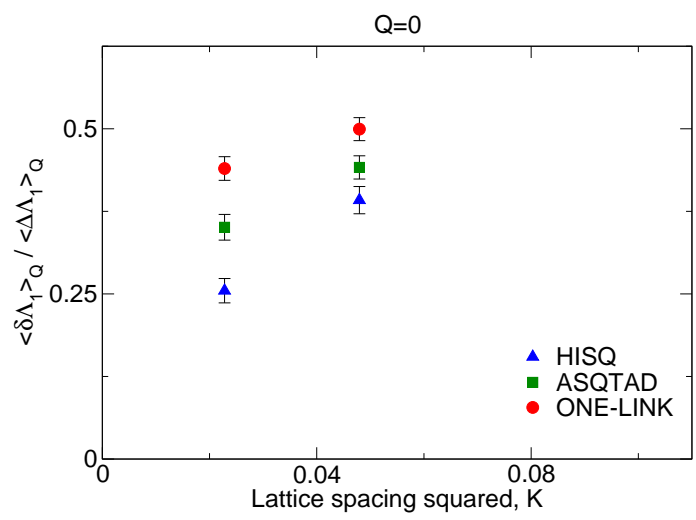

Figure 2: The ratio of the splitting to the mean of the first eigenvalue pair on the sub-ensembles with $Q=0$.

staggered action describes $N_{t}=4$ interacting copies, or "tastes", of fermions which interact via unphysical "taste-changing" interactions that vanish in the continuum limit as $a^{2}$. In the limit $a \rightarrow 0$ there is an $S U\left(N_{t}\right) \otimes S U\left(N_{t}\right)$ chiral symmetry, and the spectrum is therefore an exact $N_{t}$-fold copy of the continuum.

At sufficiently small lattice spacings we expect to see an approximation of the continuum picture. Fig. 1 shows the low-lying (positive) modes of a typical super-fine SU(2) configuration with $Q=2$. In $\mathrm{SU}(2)$ there is an additional, exact, pair-wise Kramers degeneracy in the spectrum at finite lattice spacing $[14,15]$. We only show one of each pair. As expected, we see $N_{t} Q / 4=Q$ near-zero modes with chirality near unity. The remaining modes have negligible chirality and fall into near-degenerate pairs ( $N_{t} / 2$-plets). In Fig. 2 we plot the expectation value of the splitting of the lowest pair (divided by their mean) as a function of the lattice spacing. For improved staggered actions the splitting is small and appears to be nearly proportional to $a^{2}$ for the super-fine and fine ensembles.

The Banks-Casher relation relates the spectral density

$$
\rho(\lambda) \equiv \frac{1}{V} \sum_{s} \delta\left(\lambda-\lambda_{s}\right)
$$

to the (one flavour) chiral condensate:

$$
\Sigma \equiv\langle\bar{\psi} \psi(m=0)\rangle=\frac{2}{N_{t}} \pi \lim _{\lambda \rightarrow 0} \rho(\lambda)
$$

where the prefactor corrects for the tastes and the Kramers degeneracy [14]. We plot the spectral density in Fig. 3. Even for the ONE-LINK operator the scaling is good. As we only calculate the smallest 50 eigenvalues on each configuration, the spectral density is cut off at large eigenvalues. The data is well described by a quadratic polynomial from $\lambda / \sqrt{K}=0.2$ to very near the cut-off. We show the extrapolation to zero eigenvalue and associated chiral condensate in Fig. 3. We expect a common continuum limit once the renormalisation factors, currently unknown, are included. The renormalisation is expected to be much smaller for HISQ, and the bare chiral condensate for this action is already close to the expected value. 

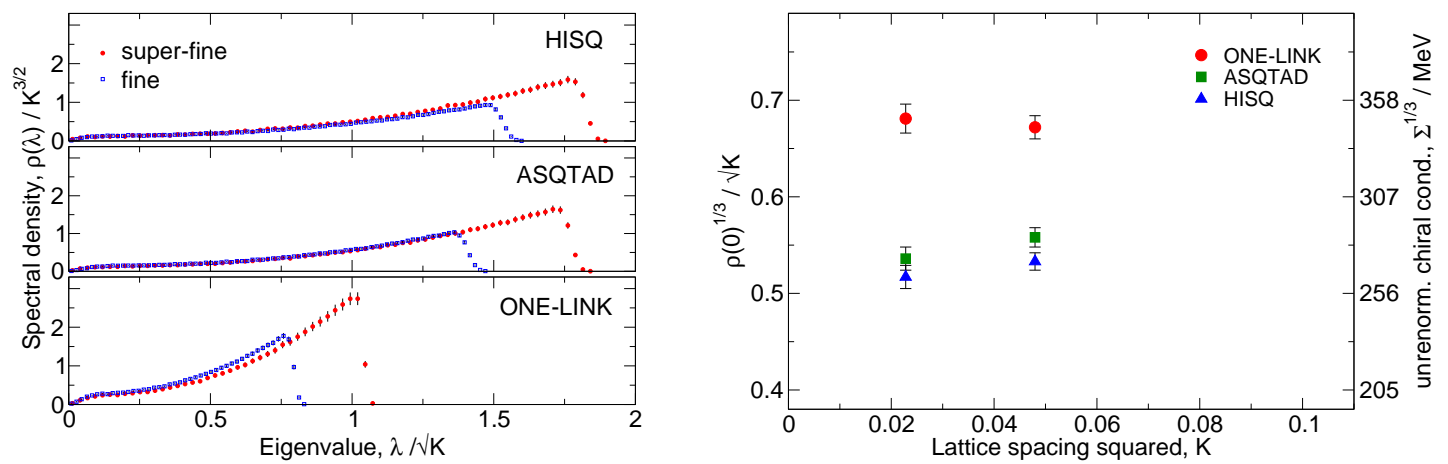

Figure 3: The spectral density, and its extrapolation to zero eigenvalue.

\section{Comparison with $\varepsilon$-regime predictions}

Our simulation volumes have been chosen to be in the $\varepsilon$-regime:

$$
\frac{1}{\Lambda_{\mathrm{QCD}}} \ll L \ll \frac{1}{m_{\pi}}
$$

i.e. the lattice size is intermediate between the Compton wavelengths of the (pseudo-) Goldstone boson(s) and the confined hadronic states. In the $\varepsilon$-regime, the distribution of low-lying modes of the massless Dirac operator in a sector of fixed topological charge can be described by a finite volume effective partition function. The predictions depend only on the underlying chiral symmetry group, the topological charge (controlling the number of zero modes) and the chiral condensate, $\Sigma$ (which sets the scale). The apparent $\varepsilon$-regime universality allows the distributions to be calculated using random matrix theory (RMT).

In the continuum limit, the eigenvalue distributions for $\mathrm{SU}(2)$ are given by the chiral Orthogonal Ensemble (chOE) [16, 17, 18, 19]. At finite lattice spacing, however, the distributions come from the chiral Symplectic Ensemble (chSE) [16, 20] and are markedly different [15]. By comparing the measured distribution of the Dirac eigenvalues to the chOE and chSE predictions, we can understand at what lattice spacing we see restoration of the full continuum chiral symmetries.

In Fig. 4 we show such a comparison for histograms of the lowest eigenvalue (rescaled by $\Sigma V$ ). The restoration of the full continuum chiral symmetry group occurs very rapidly. With ONE-LINK fermions at $a=0.10 \mathrm{fm}$ we see good agreement with the predictions from the chSE, as expected for finite lattice spacing. At $a=0.07 \mathrm{fm}$, however, HISQ eigenvalues follow the chOE distribution, as expected in the continuum limit. We have used a different value for $\Sigma$ in each case. We shall discuss the consistency of these in a future paper.

In our earlier work we saw a similar crossover in behaviour for SU(3), where the eigenvalue distributions are very similar. The lattice spacing needs to be very coarse, however, before the discrete prediction is clearly seen [21].

\section{Summary and conclusions}

We have presented some results from our ongoing study of the spectrum of improved staggered Dirac operators. From the properties of the low-lying eigenmodes we aim to understand and 

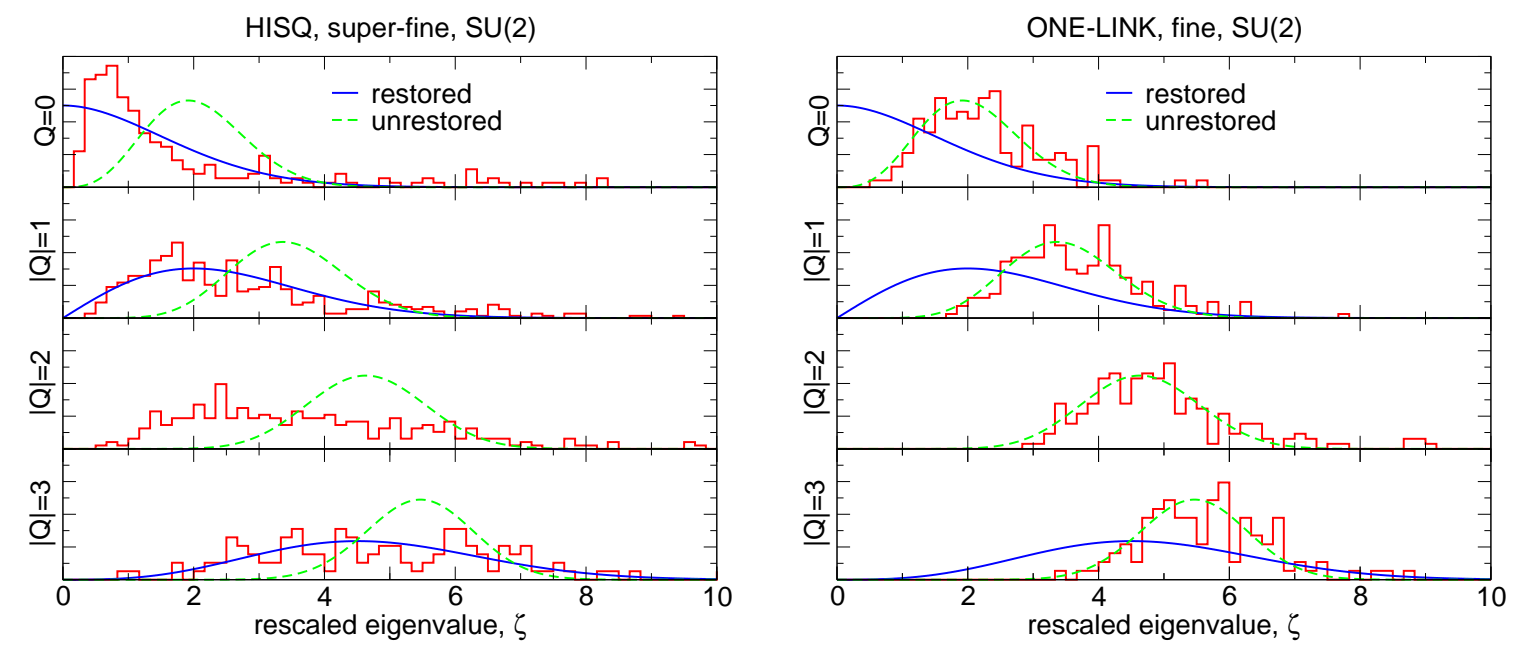

Figure 4: Comparison of the distribution of the smallest non-zero eigenvalue with $\varepsilon$-regime predictions: "restored" denotes the continuum-like chOE and "unrestored" the chSE for the discrete case.

validate the use of improved staggered fermions in realistic QCD simulations.

In these proceedings we have concentrated on the properties of the spectrum when coupled to $\mathrm{SU}(2)$ gauge fields. The motivation for using this gauge group is three-fold. First, $\mathrm{SU}(2)$ and SU(3) share a lot of important non-perturbative features. Also, SU(2) shows a remarkably close quantitative agreement with the more physical $\mathrm{SU}(3)$ in many physical quantities. Finally, the $\varepsilon$ regime eigenvalue distributions between lattice and continuum differ far more in $\mathrm{SU}(2)$ than in SU(3), giving a more sensitive probe of chiral symmetry restoration.

We have shown that the improved staggered lattice Dirac operators give a very good approximation to the Atiyah-Singer index theorem (which formally holds only in the continuum limit). The non-zero eigenvalues form near-degenerate $N_{t}$-plets as expected, with the splittings numerically small and reducing as $a^{2}$. This indicates that ASQTAD and HISQ operators have good control over the taste-changing interactions. The Banks-Casher relation appears to be satisfied. All of these results mirror our earlier findings in $\mathrm{SU}(3)$.

Lastly, we compared the distributions of the smallest non-zero Dirac eigenvalues with the $\varepsilon$ regime predictions as a probe of chiral symmetry restoration. The crossover between lattice and continuum chiral symmetry groups was seen, and occurred over a much narrower range of lattice spacings than in $\mathrm{SU}(3)$.

The results presented here show that improved staggered fermions behave exactly as expected in $\mathrm{SU}(2)$ as well as $\mathrm{SU}(3)$, and that the improvement program is successful at not only reducing mesonic splittings but also in describing the topology and chiral symmetry restoration of QCD and related theories at lattice spacings currently accessible in dynamical Monte Carlo simulations.

\section{Acknowledgments}

We thank Poul Damgaard for useful discussions, and Philippe de Forcrand for an SU(3) topology measurement code adapted by the authors for SU(2). E.F. and A.H. respectively thank the UK 
PPARC and Royal Society for financial support.

\section{References}

[1] E. Follana, A. Hart and C. T. H. Davies, Phys. Rev. Lett. 93 (2004) 241601 [hep-lat/0406010].

[2] E. Follana, Nucl. Phys. Proc. Suppl. 140 (2005) 141-147 [hep-lat/0409062].

[3] E. Follana, A. Hart, C. T. H. Davies and Q. Mason, Phys. Rev. D72 (2005) 054501 [hep-lat/0507011].

[4] E. Follana, A. Hart and C. T. H. Davies, PoS LAT2005 (2006) 298 [hep-lat/0509177].

[5] E. Follana, A. Hart and C. T. H. Davies, Nucl. Phys. Proc. Suppl. 153 (2006) 106-113.

[6] M. J. Teper, hep-th/9812187.

[7] V. G. Bornyakov, E. M. Ilgenfritz and M. Mueller-Preussker, Phys. Rev. D72 (2005) 054511 [hep-lat/0507021].

[8] HPQCD Collaboration: A. Gray et al., Phys. Rev. Lett. 95 (2005) 212001 [hep-lat/0507015].

[9] P. de Forcrand, M. Garcia Perez and I.-O. Stamatescu, Nucl. Phys. Proc. Suppl. 47 (1996) 777-780 [hep-lat/9509064].

[10] P. de Forcrand, M. Garcia Perez and I.-O. Stamatescu, Nucl. Phys. B499 (1997) 409-449 [hep-lat/9701012].

[11] M. Teper, Nucl. Phys. Proc. Suppl. 83 (2000) 146-150 [hep-lat/9909124].

[12] MILC Collaboration: K. Orginos, D. Toussaint and R. L. Sugar Phys. Rev. D60 (1999) 054503 [hep-lat/9903032].

[13] E. Follana, et al. in preparation.

[14] S. J. Hands and M. Teper, Nucl. Phys. B347 (1990) 819-853.

[15] J. J. M. Verbaarschot, hep-th/9710114. Prepared for NATO Advanced Study Institute on Confinement, Duality and Nonperturbative Aspects of QCD, Cambridge, England, 23 Jun - 4 Jul 1997.

[16] P. Forrester, Nucl. Phys. B 402 (1993) 709.

[17] R. G. Edwards, U. M. Heller, J. E. Kiskis and R. Narayanan, Phys. Rev. Lett. 82 (1999) 4188-4191 [hep-th/9902117].

[18] P. H. Damgaard and S. M. Nishigaki, Phys. Rev. D63 (2001) 045012 [hep-th/0006111]. See corrections at hep-th/0006111v2.

[19] T. Nagao and S. M. Nishigaki, Phys. Rev. D63 (2001) 045011 [hep-th/0005077].

[20] M. E. Berbenni-Bitsch, S. Meyer and T. Wettig, Phys. Rev. D58 (1998) 071502 [hep-lat/9804030].

[21] P. H. Damgaard, U. M. Heller, R. Niclasen and K. Rummukainen, Phys. Rev. D61 (2000) 014501 [hep-lat/9907019]. 